\title{
Exploring the different management structures in nurses responses and treating of patients' postoperative pain: A qualitative triangulation study
}

\author{
Manaporn Chatchumni ${ }^{1,2}$, Ampaporn Namvongprom ${ }^{3}$, Henrik Eriksson ${ }^{4}$, Monir Mazaheri ${ }^{5}$
}

${ }^{1} \mathrm{RN}, \mathrm{PhD}$, Senior Lecturer, School of Nursing, Rangsit University, Pathumthani, Thailand

${ }^{2}$ Postdoctoral Research Affiliate, Department of Health Sciences, The Red Cross University College, Stockholm, Sweden

${ }^{3} \mathrm{RN}, \mathrm{PhD}$, Assistant Professor, School of Nursing, Rangsit University, Pathumthani, Thailand

${ }^{4} \mathrm{RN}, \mathrm{PhD}$, Professor, Department of Health Sciences, The Red Cross University College, Stockholm, Sweden

${ }^{5} \mathrm{RN}$, PhD, Senior Lecturer, Department of Health Sciences, The Red Cross University College, Stockholm, Sweden

Type of article: Original

\begin{abstract}
Background: Nurses' roles are an important aspect of their approaches to pain management and monitoring in the post-operative phase of recovery in a surgical ward. A barrier to successful pain management may be the nurse's perceptions of the patient in pain, which are confounded by the patterns of communication within individual contexts. We need to study, grasp and understand the complexities of the pain management practice within the context of the surgical ward in order to be able to improve the practices and design appropriate interventions to help patients in need.

Objective: The purpose of this study was to explore nurses' postoperative pain management practices.

Methods: This qualitative triangulation study was conducted in a surgical ward at a public hospital in Bangkok (Thailand) from 2012 to 2015 . We applied four qualitative methods in the study: 1) observations in a postoperative pain management setting (100 hours); 2) in-depth interviews (12 nurses), 3) three focus group discussions (18 nurses), and 4) narratives relating to 69 critical incidents gathered during recurrent visits over a period of ten weeks (9 nurses). Content analysis, as outlined in grounded theory, was applied.

Results: The 40 nursing staff made their observations of the participants by conducting go-along interviews while they worked in the surgical field. The group of nurses comprised of 20 females and 4 males, age-ranged between 21-49 years of age, and their nursing experience ranged from 1-28 years. From our analysis, nurses verified patients' pain by using double- and triple-control methods to document and record it, thus managing pain by administrative procedures rather than being proactive in providing pain relief. Therefore, communication and information about the patients' pain and subsequent treatment of postoperative pain caused delays that may hamper the adequate use of available analgesics for pain relief. Levels of experience in communicating between nurses, other professionals, and patients were a main cause of delays in treating and managing pain.

Conclusion: The complex communication system that would improve the communication ways leads to better standards of practice and quality of care.

Keywords: Pain management, Focus groups, Grounded theory, Analgesics, Pain, Postoperative, Communication

Note: This manuscript has been organized using the Consolidated Criteria for Reporting Qualitative Studies (COREQ): 32-item checklist, developed from: Tong A, Sainsbury P, Craig J. Consolidated criteria for reporting qualitative research (COREQ): a 32-item checklist for interviews and focus groups. Int J Qual Health Care. 2007; 19 (6):349-57, DOI: 10.1093/intqhe/mzm042, PubMed PMID: 17872937.
\end{abstract}

\section{Abbreviations / Acronyms:}

COREQ: Consolidated criteria for reporting qualitative research; FGDs: focus-group discussions

\section{Corresponding author:}

Dr. Manaporn Chatchumni, School of Nursing, Rangsit University Muang-Ake, Phaholyothin Rd., Pathumthani, Thailand, Zip code: 12000. Tel: +66 (0) 86793-2079, Email: manaporn@rsu.ac.th

Received: January 03, 2019, Accepted: March 28, 2019, Published: June 2019

iThenticate screening: March 26, 2019, English editing: April 22, 2019, Quality control: April 24, 2019

This article has been reviewed / commented by three experts

Funding / research project approval: Mälardalen University (Doctoral dissertation number: 204/2016)

Ethics approval: Uppsala institutional review board number: Dnr 2012/383; Ethics approval code: 16/2555

(C) 2019 The Authors. This is an open access article under the terms of the Creative Commons Attribution-NonCommercialNoDerivs License, which permits use and distribution in any medium, provided the original work is properly cited, the use is non-commercial and no modifications or adaptations are made. 


\section{Introduction}

Pain management in a surgical ward is greatly influenced by the cultural context of nursing practices. Published studies link the quality of care provided to the patients' cultural background, such as their language, religion, and beliefs $(1,2)$. Nurses' roles are central to the assessment of symptoms and to giving advice on pain management to patients in the postoperative phase of recovery in a surgical ward (3-5). Furthermore, nurses must function collaboratively with other professionals, such as doctors, pharmacists, and physiotherapists, in a multidisciplinary team $(3,4)$. A nurse is a healthcare professional who works extremely closely with the patients in each postoperative field. Nurses applied techniques in intervention and pain management that lead to the relief of postoperative pain. Nurses must be aware of the patient's own knowledge in relation to both their medical background and their individual knowledge, and they should modify the knowledge that they provide to fit with their patient's understanding (5). It is also important for nurses to have contextual knowledge, which comprises cultural norms, beliefs and attitudes, to help them improve the care they provide in pain management practices. The published findings of other countries on the influence of nursing pain management in a surgical field, led to being effective in improving nurses' pain knowledge, attitudes, and assessment through a pain education program (4-7). In Thailand, the few studies that have explored the nursing culture in postoperative care, have described them as being routine and almost ritualized, in their approaches to pain management and monitoring in the post-operative phase of recovery in a surgical ward. These include a lack of commitment to implementing new multimodal models of care and drawing on research knowledge and evidence-based practices (8-10). This might explain why these nurses provide insufficient pain management to postoperative patients in hospital. Therefore, the culture of nurses in pain management may play a crucial role in postoperative pain management. The specific objectives of the study were: 1) to explore how nurses assess and manage postoperative pain in surgical ward, 2) to explore the characteristics of nursing care that contribute to the successful and unsuccessful practices in the nurses' experiences of being situated in the postoperative pain management phase on a surgical ward, and 3) and to explore nurses' postoperative pain management practices.

\section{Material and Methods \\ 2.1. Research design}

The study employed a methodological triangulation design. Methodological triangulation is a research approach that uses a variety of different data collection methods, and can include secondary data analyses drawing on qualitative data from earlier studies, and other data collected using multi-method triangulation (11-14). Using an alternative qualitative research design to pursue the overall aim of the study adds to its richness. There is knowledge to be gained from engaging in observations and participating with the nurses to explore their approach to assessing and managing patient's pain during the postoperative phase. Understanding the cultural context and having an awareness of cultural sensitivities involves exploring the nurse-patient relationship, their ways of communicating, and the patients' pain-related health behaviours, as many such situations can be encountered in daily practice (11-15).

\subsection{Data collection}

The data collection took place in a surgical ward at a public hospital in Bangkok, Thailand (the authors removed the name of the hospital as this might be a risk to potential violation of confidentiality of the data and disclosure of the participants; however, the journal has received the required confirmed information). The data collection took place in a surgical ward at a public hospital in Bangkok, Thailand. Data were collected during 2012-2015, and conducted according to the consolidated criteria for reporting qualitative research (COREQ) as the 32 - item checklist for interviews and focus groups recommended by Tong and colleagues (16). Qualitative methods are especially useful when exploring complex phenomena, and the variety of chosen approaches here, may also offer new ways of examining the nurses' experiences of managing pain. The following approaches were used in this study: 1) making observations of the participants (and conducting go-along interviews) while they worked in the surgical field for a total of 100 hours $(17), 2$ ) focus group interviews with 18 nurses, who were divided into three groups of focus-group discussions (FGDs) (18), 3) in-depth interviews (12 nurses) (19), and 4) critical incident interviews with 9 nurses during recurrent visits over a period of ten weeks that collected 69 critical incidents (20). The nurses were chosen in accordance with theoretical sampling as Benner's nursing theory (21) was used for recruiting participants to the FGDs, in-depth interviews and critical incident interviews. Benner (21) identified that there are five levels of nursing competency. These levels were used to guide the sampling in this study, and nurses were categorized as: novice, when nurses had 1-3 years of working experience; advanced beginners, when nurses had 4-5 years of working experience; and an expert level of competency, when nurses had more than 10 years of working experience. However, of our participants, no one had 6-9 years of working experience to a level of proficient competency. Details on participant characteristics are illustrated in Table 1. 
Table 1. Participant characteristics

\begin{tabular}{|l|l|l|l|l|}
\hline Variable & Observations & FGDs & Interviews \\
\hline Gender & Female & 20 & 17 & 15 \\
\hline & Male & 4 & 1 & 6 \\
\hline Age & Range (year) & $21-49$ & $21-55$ & $22-50$ \\
\hline & Median (year) & 25 & 40 & 38 \\
\hline Nursing experience (year) & $1-28$ & $1-17$ & $2-29$ \\
\hline
\end{tabular}

\subsection{Data analysis}

An inductive approach was employed in conducting the analyses accordance triangulation of data from different sources and times $(13,14)$. The sequence of the analyses was determined based on the type of data sources, including the participants and the collection method, and then analyzed: first, the observations and participatory data; second, the FGDs; and third, the interviews. Afterwards, all of the observations and interviews were analyzed together to deepen understanding within the postoperative pain management practice. The data analysis method was determined based on the number of participants and the data collection method of each data source. Triangulation of data was used to analyze the multiple data sources within the data analysis process, which consisted of three phases, and using coding elements guided by the observation, FGDs, and interviews $(13,14)$. Researchers used an inductive method in the first instance, then modified it during the analysis process as well as ensued methods according to a trustworthiness aspect of qualitative research: credibility, transferability, dependability, and confirmability $(22,23)$. The authors familiarized themselves with the data by performing analysis continuously throughout each study period. The analysis was combined and discussed by each of the authors until consensus was reached.

\subsection{Ethical consideration}

The studies were approved by the ethics committee of a public hospital in Thailand (Code: 16/2555). All participants received written information and were fully informed about the aim and design of the study before giving their consent. The participation in this study was voluntary and the participants had the right to exit the study at any time without any question. In addition, they were assured of our policy about the confidentiality of their personal information.

\section{Results}

The nurses' responses to the patients in pain were characteristically hindered by cultural issues that influenced the nursing practices. For example, instead of being proactive, they waited for the patients to ask for help, which is a negative element in pain management practices. Being able to influence and control pain is integral to effective postoperative pain management, and being able to recognise the patient in pain and engage with the patient to make pain relief available when the patient needs it is a vital element of the nurse-patient relationship. The nurses' system for responding to a patient in pain was a complex network of communication to assess and manage pain in their practices. The nurses were employed to assess and record pain by using the multiple methods of double / triple control, after which pain management was based on the prescription of pain medication by a physician, which often caused delays. Pain management was also influenced by the nurses' culture in managing pain, which was influenced by their own experiences of controlling pain and by their engagement in the nurse-patient relationship by promoting trusting and supportive relationships, as well as by the availability of treatment and nursing care when needed.

\subsection{Nursing culture in assessing pain scores and managing pain}

The nursing practices in assessing pain and managing pain were influenced by their own perceptions of the patients in pain and of pain management. In addition, the essence of Thai-ness in Thai culture was particularly influential and encompassed both pain assessment and pain management. For instance, kreng jai is a typical feature of Thai culture that relates to the idea that the patient has consideration for the nurses' and the physicians' feelings, so they often avoid asking them to treat their pain, despite it having high intensity. For example:

As I observed, the nurse stayed with their patient just as long as it would take to wait for an analgesic injection. Yet, she only gave two paracetamol tablets $(500 \mathrm{mg})$ to her patient while telling him that, if he has unrelieved pain, 'You could tell me again, I will give an analgesic injection to you according the physician's order.' (A 48-year-old female nurse with 25 years' experience)

'I was just talking to one patient who had bladder cancer ... and was experiencing a lot of pain and his physical expression were to writhe in agony on the bed. I would just try to tell him that he should try to meditate, as I knew, from his background, he had been ordained as a monk, so I just explained and convinced him to keep practising meditation one more time. But after that, I visited him at his bedside, and 
he looked uncomfortable. Unfortunately, his bladder was full, and made him feeling pain and discomfort. So, the physician decided to re-operate to resolve his complication of bladder cancer. (Observation Interviews, A 48-year-old female nurse with 25 years' experience)

The routines of pain assessment involve monitoring pain within the assumption that pain is the fifth vital sign. However, the nurse had distinctly interpreted this patient's pain intensity and predicted that the patient's tolerance level was greater than that of others in the nurse's experience. These pain assessment skills differ with the expert level of competency in that they are more developed than those at a novice nurse's level or those of nurses' aides. For this reason, it is important to develop pain assessment skills to provide effective pain management practice.

"In the past I used numerical scores, the tool with the faces scale and the numerical scale, but now they have disappeared or been destroyed. Mostly, I find that nurses' aides make distinct assessments, which often they only ask their patient while checking their vital signs, such as when the nurses' aides ask, "Do you feel any pain and what is your pain score?" I understand that some are educated differently, and that they just do it as a routine, then I have to check again, when the nurses' aides tell me about some of the patients who were in pain and who scored ten. So, then I have to go back and see the patient and assess his pain again before I can give him his medication. Perhaps, the nurses' aides were not clear when explaining the pain scores to the patient. (In-depth interview: A 38-year-old male nurse with 15 years' experience)

\subsection{Responses to patient-in-pain system}

The nurses experienced difficulties in managing postoperative pain as a consequence of the complexity in the system of communication in responding to the patient's pain. Within this system, the nurses use double- and triplecontrol methods, of pain assessment tools, to enable them to assess the intensity of their patients' pain. Furthermore, the nurses must communicate with the whole care team through the relaying of documents and records to carry out effective treatment. Although the nurses' efforts to communicate levels of pain were often missing within the patients' self-reported pain, the nurses relied on their own experiences in pain assessment.

In my experience with pain assessment, perhaps, his pain score is eight, but he can manage to sleep well. The pain score is recorded on the graph sheet, so, I have seen the score points on it and the pain sheet covers information about the patient in pain, like a record of how many pain scores and what kind of pain medication. Sometimes, this document does not record that completely, however, we must plot pain scores on the graph sheet every 4 hours. I guess that it could be that, for the patients in pain, it is quite a good method for assessment after we give pain medication. If the pain scores of the patients do not decrease, then I will notify the physicians or consult with the team members about what to do for the patient to relieve his pain. (In-depth interview: A 49-year-old female nurse with 28 years' experience)

Here, the nurse was responding to the patient's discomfort and pain: the impact of the psychological responses (such as crying, anger, grinding teeth and jaw clenching) to pain. Many of the participants perceived that the patient's psychological response to pain worked together with their emotions and behaviors, and could potentially cause a risk to the patients, as one informant expressed:

In my own thoughts, the pain causes more discomfort when the patient has tissue injuries, which causes psychological effects. Such as the patient's mood has changed and their behaviour changes too, while someone else might express themselves by crying and they do not want to change positions. (FGDs: A 38year-old female nurse with 15 years' experiences, G1)

By observing actual situations where pain assessment and pain management was practised in postoperative care, and by conducting go-along interviews, we found that nurses were influenced by their own knowledge and beliefs, which were often culturally sensitive. The actions of the more experienced nurses, by laying down the foundations of nursing culture, allowed their own knowledge to influence nursing practices, particularly in relation to their experiences of the patient in pain, their perception of discomfort, and how this leads to restricted mobility and changes in mood. The nurses explained that they avoided complying with the patient's self-reported pain intensity in their pain assessment and pain management approaches, and instead, responded to this pain by determining patients' tolerance for pain in accordance with their own experiences. By adopting this strategy, they might be missing an opportunity to consider their patient's own experience. The reasons for delays in treating pain and providing nursing care was that they relied on a multiple method of communication, in using multiple documents to record and report 
pain, and various methods of scoring of pain intensity, which meant that the implementation of measuring their patient's pain was often insufficient or inappropriate in pain management practice.

\subsection{Involving personal experiences to assess pain and managing pain}

Within the FGDs with nurses, they revealed that they are allowed to treat their patients' pain by using prescription medication, which involves relying on their own experiences of pain management. The nurses who were observed carrying out pain management were often likely to wait for their patient to request pain medication rather than ask the patient. They did not apply pain management strategies to assess their patient's pain; instead, they assessed the patient's level of pain and engaged in their practices based on their anecdotal experiences in caring for patients. The patients should be encouraged to articulate their own experiences of pain, and nurses should be allowed to prioritise their care to respond to their patients' needs. However, to actively succeed in managing pain at the nurse's discretion, such engagement with the patient must also be addressed within the nurse-patient relationship and associated methods of communication. The nurses should respond positively by providing appropriate medication and nursing care when patients report that they are in pain, and they should trust the patient's voice and their responses to the self-report tool, which is a more accurate assessment of pain.

In my experience of having my own surgery, if the patient admits to having pain, stating "Oh! It's so painful," it is easy to understand and I know that it is very painful. If the patient requests pain medication, then I must give medication according to the physician's orders because the patient will recover earlier, which will enable them to leave the hospital bed sooner. In contrast, if the patient's pain is unrelieved for a long period of time, it is difficult to resolve. So, I must share information with the nurses' aides, to allow time to arrange medication for patients' surgery or for the patients with pain through cancer, who require pain medication. (Interview-1: A 48-year-old female nurse with 25 years' experience)

It is crucial that treatment for pain and nursing care are available when needed, and that the nurse's ability to adopt pain management approaches encompasses Thai culture. For instance, Mee nam jai relates to all people helping one another, but also to the nurse being willing to help their team members, even when they do not request help.

One patient was in the third day following cyst excision in the intestine. At first, he felt pain, then I gave morphine, $3 \mathrm{mg}$, around the clock. On the second day, he was a lot better, and his wound had reduced pus, so I gave him paracetamol and that was enough for him. If necessary, I would have used injected pain medication. Today, he is better and can perform activities by himself. (Interview-3: A 23-year-old male nurse with 3 years' experience)

This indicates that a powerful level of engagement exists between the nurses and the patients. It is important to nursing practice that the strategies they adopt should be conducive to building a trustful relationship with the patient and their relatives and to provide information about pain relief and the recovery phases for patients in postoperative care. The most important issue in the control of pain in postoperative phases is that pain medication and nursing care should be made available when the patient needs it. The nurse can successfully control the patient's pain intensity when they need it by administering the prescribed dose, but they first need a standing order to be raised by the physician (i.e., a morphine injection every four or six hours in the 24 to 48 hours after surgery), and this is often raised only after they have been alerted by the complex and time-consuming reporting system.

\section{Discussion}

Our results indicated that the system of nurses' pain management in daily practice involves the nurses' perceptions of the patient in pain, methods of assessing pain, and that the nursing approaches adopted to manage pain management vary according to the individual nurse. Furthermore, the findings show that "pain management" is a system of different structures in which nurses' maneuvers handle patient's pain. Moreover, the findings included indications that the nurses employed reflective practice, which was emphasized and understood as being about how the Thai culture and nursing culture influenced their practices on a day-to-day basis in the postoperative ward. As similar to previous studies, we found that the cultural aspects of nursing impact on pain management practices including cultural and religious aspects related to 'Thainess', Buddhism, the nursing profession and nurse-patient, doctor-patient relationships $(8-10,19)$. Issues in the communication of information about the patients' pain and subsequent treatment of postoperative pain were identified in some part to cause delays and may hamper the adequacy of the use of available analgesics for pain relief. Cultural sensitivities within the Thai context sometimes hindered the interaction between the nurses and the patients, whereas, had they engaged their ability to foster trust between the nurses and patients, this might instead have led to successful pain management when the patients needed it most (Figure 1). 


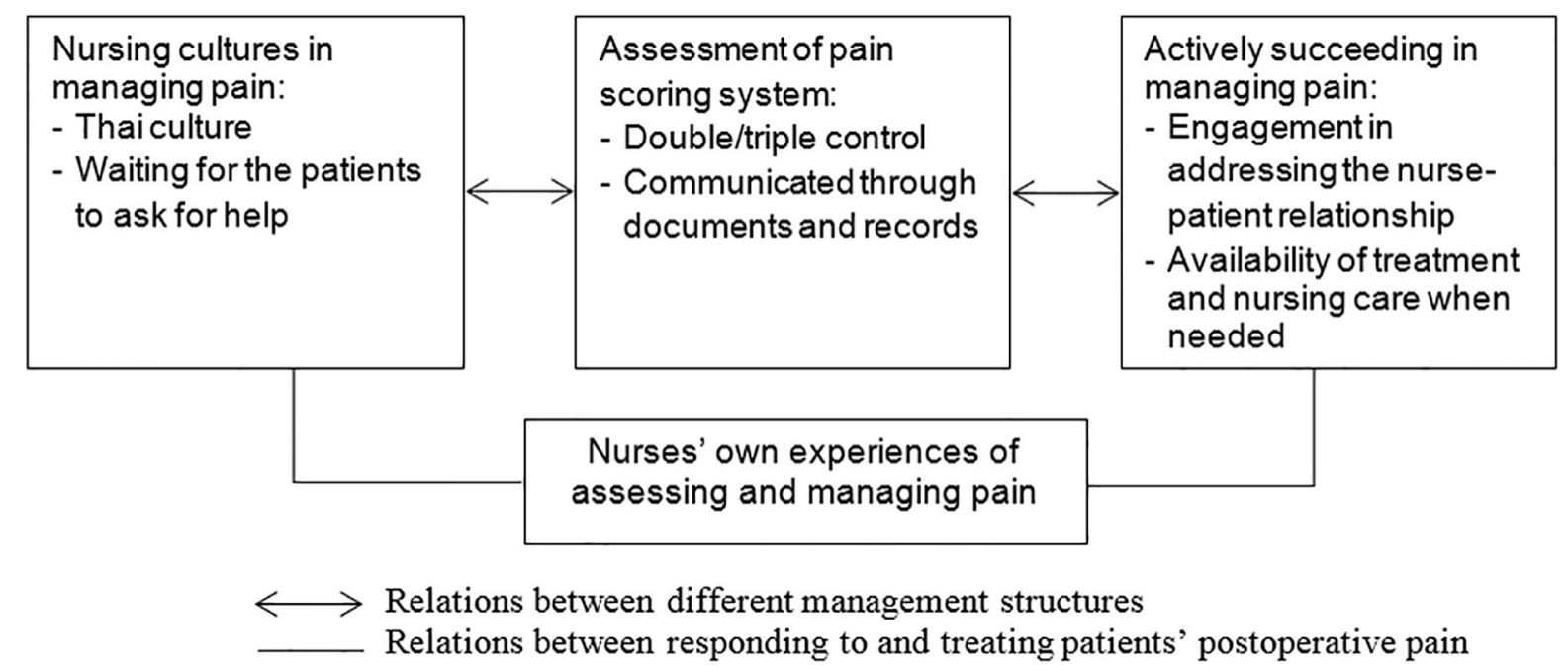

Figure 1. Responding to and addressing patients' postoperative pain system model

The "Responding to and addressing patients' postoperative pain system model" reveals how the overlapping management systems interact and are dependent on each other. As reflected in the focus group discussions, the lack of knowledge of nursing skills was identified as a main element of causing delays in treating the patients' pain, and this was caused mostly by the complexity of the communication, that is to say, the interaction between nurses' aides, nurses, physicians, and patients in pain. In the last few decades, pain management guidelines have changed significantly for hospitals achieving accreditation by The Royal College of Anesthesiologists of Thailand and the Thai Association for the Study of Pain (24). One of the indicators of these guidelines was that pain levels are defined as the fifth vital sign and that these are to be assessed every four hours for postoperative patients in a surgical setting, however, the guidelines do not provide qualified means for measuring the quality of managing pain for patients in pain $(8,10,17,25)$.

Nurses act as coordinators in responding to patients in pain within a system that provides information, direction and support by using double- and triple-control methods which are mostly concerned with generating documents and records to support the patient's pain relief $(17,20)$. These findings support previous evidence in pain assessment knowledge of the quality of care that is needed to support improved outcomes for patients who are in postoperative recovery (27). Further, nurses must seek to exploit their own clinical experience, patient values, and the best research evidence in the decision-making process to help relieve their patients' pain (26). Effective pain assessment and pain management should extend to the patients' demands, and their levels of pain should be evaluated comprehensively. The subjective nature of pain means that it must not only be treated based on numerical evidencebased guidelines, but also within the patient's own preferences and cultural context (25-27). For instance, an evaluation strategy would require an awareness of the nursing approach by listening to the patients' experiences about their pain and experiences of pain care within the context and depending on the religious and cultural perspective and the pre-existing social attitudes $(5,23,28)$.

Although the nurses' perceptions of their patients' pain were demonstrated appropriately in relation to pain as being discomfort, restricted mobility and changed mood, the nurses delayed responding to their patients in pain. This delay was mostly caused by the processes of responding to pain; the nurses' aides asked the patient for numerical pain scores, they wrote down the scores, plotted them on the graph, and then recorded them in a document before the nurses could respond $(18,26)$. It was not clear why the nurses waited for the patients to ask for help, but we found that the nurse-patient relationship often focused on the impact of the presence of the patient's relatives to help in communicating. Reflective practices helped the nurses to consider how cultural sensitivities might be hindering the interaction between the nurses and the patients, whereas if they engaged their abilities to build trust between the nurses and patients, this might lead to achieving successful pain management when the patients need it $(6,9,10,15$, 29). Communication is vital in the nurse's role and is essential to collaborating with other healthcare professionals and their patients, particularly in relation to improving the overall outcomes of their approaches to the practice of pain management $(17,19,30)$. 


\section{Conclusions}

In summary, this study showed that pain management is not one, but rather several complex structures, in which nurses' maneuvers vary in handling patient's pain. Nurses' approaches to managing these structures create pain management protocols, which undoubtedly will vary among the individual nurses towards the patients. To illustrate these structures, this study presented a "Responding to and addressing patients' postoperative pain system model". By linking culture, experiences, a scoring system and nurses managing strategies, a richer understanding can be gained regarding the complexity of pain management. The study also gives some indications of why pain management sometimes does not live up to the standards expected from patients and intentions in policies. We recommend that policymakers, educational and clinical institutions need to address not only the different management structures that exist regarding pain management, but also how they are connected as we have presented in this model. These findings can serve to challenge existing guidelines and facilitate the development of new nursing guidelines and/or policies in pain management. It is therefore important to bring about a change in nursing culture that in turn will influence the protocol process, the cost of providing healthcare, and improvement trends in the surgical field, such as decreasing complications, decreasing the average length of stay and meeting the increasing demands of flexibility and efficiency that patients need.

\section{Acknowledgments:}

The first author's postgraduate Ph.D. thesis from Mälardalen University (Uppsala institutional review board number: Dnr 2012/383, Doctoral dissertation number: 204/2016). The authors wish to thank all the nurses who participated in this study. We also wish to acknowledge specifically and thank Rangsit University for the grant to support this study and wish to thank Mälardalen University for their help and support.

\section{Conflict of Interest:}

There is no conflict of interest to be declared.

\section{Authors' contributions:}

$\mathrm{MC}, \mathrm{HE}$, and MM conceptualized and designed the research project. MC performed the data collection. All authors contributed to the analysis or interpretation of data. MC and AN drafted the manuscript. MC, HE, and MM revised the manuscript. All authors provided significant input in the manuscript, and read and approved the final version of it. All authors are accountable for all aspects of the work in ensuring that questions related to the accuracy or integrity of any part of the work are appropriately investigated and resolved.

\section{References:}

1) Booker SQ. African Americans' perceptions of pain and pain management: A systematic review. J Transcult Nurs. 2016; 27(1): 73-80. doi: 10.1177/1043659614526250. PMID: 24841472.

2) Paradies $Y$, Truong M, Priest N. A systematic review of the extent and measurement of healthcare provider racism. J Gen Intern Med. 2014; 29(2): 364-87. doi: 10.1007/s11606-013-2583-1. PMID: 24002624 PMCID: PMC3912280.

3) Wikström L, Eriksson K, Årestedt K, Fridlund B, Broström A. Healthcare professionals' perceptions of the use of pain scales in postoperative pain assessments. Appl Nurs Res. 2014; 27(1): 53-8. doi: 10.1016/j.apnr.2013.11.001. PMID: 24387871

4) Zhang CH, Hsu L, Zou BR, Li JF, Wang HY, Huang J. Effects of a Pain Education Program on Nurses' Pain Knowledge, Attitudes and Pain Assessment Practices in China. J Pain Symptom Manage. 2008; 36(6): 616-27. doi: 10.1016/j.jpainsymman.2007.12.020. PMID: 18599261.

5) Aziato L, Adejumo O. An ethnographic exploration of postoperative pain experiences among Ghanaian surgical patients. J Transcult Nurs. 2015; 26(3): 301-7. doi: 10.1177/1043659614526246. PMID: 24797253.

6) Chambers T, Shepler J. Barriers to adult surgical pain management. J Pain. 2018; 19(3), S53. doi: 10.1016/j.jpain.2017.12.126.

7) Al-Hashimi M, Scott S, Griffin-Teall N, Thompson J. Influence of ethnicity on the perception and treatment of early post-operative pain. Br J Pain. 2015; 9(3): 167-72. doi: 10.1177/2049463714559254. PMID: 26516573, PMCID: PMC4616978.

8) Maneewat K. Nursing care practices and workplace relations in a Thai surgical ward: An exploration of clinical decision-making. 23rd International Nursing Research Congress. 2010. Available from: http://www.nursinglibrary.org/vhl/handle/10755/243190.

9) Chinnawong T. The influences of Thai Buddhist culture on cultivating compassionate relationships with equanimity between nurses, patients and relatives: A grounded theory approach. 2007. $\mathrm{PhD}$ thesis, Southern Cross University, Lismore, NSW. Available from: https://epubs.scu.edu.au/theses/374/. 
10) Burnard $P$, Naiyapatana $W$. Culture and communication in Thai nursing: A report of an ethnographic study. Int J Nurs Stud. 2004; 41(7): 755-65. doi: 10.1016/j.ijnurstu.2004.03.002. PMID: 15288798.

11) Shih FJ. Triangulation in nursing research: Issues of conceptual clarity and purpose. J Adv Nurs. 1998; 28(3): 631-41. doi: 10.1046/j.1365-2648.1998.00716.x. PMID: 9756233.

12) Thurmond VA. The point of triangulation. J Nurs Scholarsh. 2001; 33(3): 253-8. doi: 10.1111/j.15475069.2001.00253.x. PMID: 11552552.

13) Flick U. Triangulation in qualitative research. A companion to qualitative research. Sage Press. 2004: 17883.

14) Joslin R, Müller R. Identifying interesting project phenomena using philosophical and methodological triangulation. IPMA. 2016; 34(6): 1043-56. doi: 10.1016/j.ijproman.2016.05.005.

15) Carney M. Influence of organizational culture on quality healthcare delivery. Int J Health Care Qual Assur. 2011; 24(7): 523-39. doi: 10.1108/09526861111160562. PMID: 22204086.

16) Tong A, Sainsbury P, Craig J. Consolidated criteria for reporting qualitative research (COREQ): a 32-item checklist for interviews and focus groups. Int J Qual Health Care. 2007; 19(6): 349-57. doi: 10.1093/intqhe/mzm042. PMID: 17872937.

17) Chatchumni M, Namvongprom A, Eriksson H, Mazaheri M. Thai Nurses' experiences of post-operative pain assessment and its' influence on pain management decisions. BMC Nurs. 2016; 15: 12. doi: 10.1186/s12912-016-0136-8. PMID: 26933384, PMCID: PMC4772523.

18) Chatchumni M, Namvongprom A, Sandborgh M, Mazaheri M, Eriksson H. Nurse's Perceptions of Patients in Pain and pain management: A focus group in Thailand. Pac Rim Int J Nurs Res Thail. 2015; 19(2): 16477.

19) Chatchumni M, Namvongprom A, Eriksson H, Mazaheri M. Treating without seeing: Pain management practice in a Thai context. Pain Res Manag. 2016; 2016:9580626. doi: 10.1155/2016/9580626. PMID: 28044071, PMCID: PMC5156806.

20) Chatchumni M, Namvongprom A, Eriksson H, Mazaheri M. Engagement and availability in shaping nurses' management of postoperative pain: a qualitative study. Electron Physician. 2018; 25; 10(8): 7235 42. doi: 10.19082/7235. PMID: 30214707, PMCID: PMC6122866.

21) Benner PE. From Novice to Expert: Excellence and Power in Clinical Nursing Practice. Upper Saddle River, NJ: Prentice Hall; 2001.

22) Guba EG, Lincoln YS. Competing paradigms in qualitative research. In The Landscape of Qualitative Research. Sage. Thousand Oaks, CA. 1998: 195-222.

23) Korstjens I, Moser A. Series: Practical guidance to qualitative research. Part 4: Trustworthiness and publishing. Eur J Gen Pract. 2018; 24(1): 120-4. doi: 10.1080/13814788.2017.1375092. PMID: 29202616.

24) Royal College of Anesthesiologists of Thailand \& Thai Association for the Study of Pain. Clinical Guidance for Management of Acute Postoperative Pain. 2011. Available from: http://www.tasp.or.th/wpcontent/uploads/2017/02/POSTOPERATIVE_PAIN_2554.pdf.

25) Al Samaraee A, Rhind G, Saleh U, Bhattacharya V. Factors contributing to poor post-operative abdominal pain management in adult patients: a review. Surgeon. 2010; 8(3): 151-8. doi: 10.1016/j.surge.2009.10.039. PMID: 20400025.

26) Yin HH, Tse MM, Wong FK. Systematic review of the predisposing, enabling, and reinforcing factors which influence nursing administration of opioids in the postoperative period. Jpn J Nurs Sci. 2015; 12(4): 259-75. doi: 10.1111/jjns.12075. PMID: 25781037.

27) Ruben MA, van Osch M, Blanch-Hartigan D. Healthcare providers' accuracy in assessing patients' pain: A systematic review. Patient Educ Couns. 2015; 98(10): 1197-206. doi: 10.1016/j.pec.2015.07.009. PMID: 26223850.

28) Pain T, Kingston G, Askern J, Smith R, Phillips S, Bell L. How are allied health notes used for inpatient care and clinical decision-making? A qualitative exploration of the views of doctors, nurses and allied health professionals. Health Inf Manag. 2017; 46(1): 23-31. doi: 10.1177/1833358316664451. PMID: 27574187.

29) van Dijk JF, Schuurmans MJ, Alblas EE, Kalkman CJ, van Wijck AJ. Postoperative pain: knowledge and beliefs of patients and nurses. J Clin Nurs. 2017; 26(21-22): 3500-10. doi: 10.1111/jocn.13714. PMID: 28042880.

30) Powell R, Scott NW, Manyande A, Bruce J, Vogele C, Byrne-Davis LM, et al. Psychological preparation and postoperative outcomes for adults undergoing surgery under general anaesthesia. Cochrane Database Syst Rev. 2016; 5: CD008646. doi: 10.1002/14651858.CD008646.pub2. PMID: 27228096. 